\title{
Hypertriglyceridemic-Waist Phenotype is a Useful Global Assessment Tool for Predicting Acute Myocardial Infarction
}

Grace M. Egeland ${ }^{1,2 *}$, Jannicke Igland ${ }^{2}$, Ottar Nygard ${ }^{3,4}$, Gerhard Sulo $^{2}$ and Grethe S. Tell ${ }^{1,2}$

${ }^{1}$ Division of Health Registries, Norwegian Institute of Public Health, Bergen, Norway

${ }^{2}$ Department of Global Public Health and Primary Care, University of Bergen, Bergen, Norway

${ }^{3}$ Department of Clinical Science, University of Bergen, Bergen, Norway

${ }^{4}$ Department of Heart Disease, Haukeland University Hospital, Bergen, Norway

\begin{abstract}
Background: There has been growing research interest in exploring the utility of the hypertriglyceridemicwaist phenotype as a predictor of cardio-metabolic risk. To date, prospective studies evaluating the phenotype as a predictor of cardiovascular disease have provided variable results.

Objectives: To evaluate the usefulness of the hypertriglyceridemic-waist phenotype as an independent predictor of acute myocardial infarction beyond classical cardiovascular disease risk factors in a homogeneous Norwegian population.

Methods: Norwegian health survey (Cohort Norway) participants $(n=116,111)$, free of heart disease in 19942003, were followed through 2009 by record linkages to The Cause of Death Registry and hospital discharge diagnoses through the Cardiovascular disease in Norway project. Cox proportional hazards analyses adjusted for conventional risk factors.

Results: During a mean follow-up of 11.5 yrs $(S D=2.8), 3,624$ participants developed an acute myocardial infarction. Prevalence of an enlarged waist $>102 \mathrm{~cm}$ for men, and $>88 \mathrm{~cm}$ for women) increased from the lowest to highest quartile of triglycerides for men $(4.9 \%$ to $22.5 \%)$ and women $(6.5 \%$ to $42.1 \%$; P for trend $<0.01)$. The presence of an enlarged waist and elevated triglyceride $(>1.7 \mathrm{mmol} / \mathrm{L})$ was associated with a hazard ratio for acute myocardial infarction of $1.68(95 \% \mathrm{Cl} 1.48-1.90)$ for men and $1.95(95 \% \mathrm{Cl} 1.66-2.29)$ for women compared to those with normal waist and triglyceride level after adjusting for age, smoking and time since last meal. However, $75 \%$ of the excess risk was mediated by HDL and nonHDL cholesterol.
\end{abstract}

Conclusions: The phenotype is a useful global assessment tool but of limited value when conventional risk factors are available. Patients presenting with the phenotype should be targeted with lifestyle interventions and referred for clinical follow-up.

Keywords: Hypertriglyceridemia; Central obesity; Cardiovascular disease

Abbreviations: AMI: Acute Myocardial Infarction; CONOR: Cohort Norway; CVD: Cardiovascular Disease; CVDNOR: Cardiovascular Disease in Norway Project; ICD: International Classification of Diseases; HDL-C: High-Density Lipoprotein Cholesterol; HR: Hazard Ratio; LDL-C: Low-Density Lipoprotein Cholesterol

\section{Introduction}

The presence of hypertriglyceridemia with an elevated waist circumference, referred to as the hypertriglyceridemic-waist phenotype, is considered a proxy indicator of visceral fat which is now recognized to have greater metabolic consequences than that attributed to subcutaneous fat alone [1]. The phenotype has also been proposed to be a simpler alternative to the metabolic syndrome, and there has, therefore, been growing research interest in exploring the utility of the hypertriglyceridemic-waist phenotype as a predictor of cardiometabolic risk [2]. Numerous studies have identified the phenotype as being a strong associate and determinant of insulin resistance and diabetes in cross-sectional and prospective studies [3-7]. While a few prospective studies have evaluated the association between the phenotype and cardiovascular disease (CVD), the results of these studies are highly variable [8-12]. Thus, the usefulness of the phenotype as an independent predictor of CVD beyond classical CVD risk factors is not yet established.

In addition, data suggest that non-fasting triglycerides correlate strongly with post-prandial remnant lipoproteins, and that non-fasting triglycerides are superior to fasting triglycerides in characterizing the usual post-prandial state and its' associated risks $[13,14]$. Also, it is the remnant lipoproteins associated with elevated triglycerides and not the triglycerides, per se, that are considered atherogenic $[15,16]$. The presence of an enlarged waist with elevated triglyceride levels could identify individuals unable to clear and store excess triglycerides from over-nutrition and, therefore, identify individuals who are more likely to be chronically exposed to elevated remnant lipoproteins than individuals without the phenotype [1].

Given the strong association of the hypertriglyceridemic-waist phenotype with insulin resistance and that elevated non-fasting triglycerides associate with greater exposures to atherogenic remnant lipoproteins, we evaluated, prospectively, the risk of incident acute

*Corresponding author: Egeland GM, Department of Global Public Health and Primary Care \& Norwegian Institute of Public Health, Kalfarveien 31, N-5018 Bergen, Norway, Tel: +47 53204065; E-mail: g.egeland@igs.uib.no

Received June 19, 2015; Accepted July 06, 2015; Published July 08, 2015

Citation: Egeland GM, Igland J, Nygard O, Sulo G, Tell GS (2015) Hypertriglyceridemic-Waist Phenotype is a Useful Global Assessment Tool for Predicting Acute Myocardial Infarction. J Cardiovasc Dis Diagn 3: 207. doi:10.4172/2329-9517.1000207

Copyright: @ 2015 Egeland GM, et al. This is an open-access article distributed under the terms of the Creative Commons Attribution License, which permits unrestricted use, distribution, and reproduction in any medium, provided the original author and source are credited. 
myocardial infarction (AMI) by the hypertriglyceridemic-waist phenotype in a large population-based cohort in Norway.

\section{Material and Methods}

Participants in at least one population-based regional health screening of Cohort Norway (CONOR) between 1994 and 2003 formed the baseline of the prospective study, details of which are described elsewhere $[17,18]$. Of a total of 309,742 invitations for CONOR study participation, 59\% $(181,891)$ participated, of whom 173,243 men and women attended at least one health screening. Participants with a selfreported history of heart disease, stroke, angina, and current or past use of antihypertensive medications or with missing baseline health information $(n=32,453)$ were excluded, leaving a total of 140,790 participants for analyses. Missing data for covariates was low for the majority of parameters $(<1 \%$ for smoking, lipids, and blood pressure, $4.9 \%$ for time since last meal, and $8.8 \%$ for alcohol consumption), but waist circumference measures were missing for $17.4 \%$ of the study population as it was not included in one regional survey. After exclusion of participants with missing data, 116,111 participants had a baseline assessment of non-fasting triglycerides and waist circumference.

An 11-digit personal identifier was used to identify status of CONOR participants as of December 31, 2009 through records linkages with the Cause of Death Registry and national hospital discharge diagnoses which were obtained through the Cardiovascular Disease in Norway Project (CVDNOR), 1994-2009 [18-20]. Only 0.8\% of the cohort was lost to follow-up due to migration. The outcome for the current analyses was hospitalization or death attributed to the first registered AMI occurring between baseline through 2009 using the International Classification of Diseases (ICD) codes of 410 (ICD-9) and I21 and I22 (ICD-10). Informed consent was obtained from each participant and the study protocol conforms to the ethical guidelines of the Declaration of Helsinki as reflected in a priori approval by the Regional Ethics Committee which approved both the baseline survey and the prospective record linkages.

Non-fasting triglycerides, total and HDL-C were measured by an enzymatic method (Boehringer 148393. Boehringer-Mannheim, Federal Republic of Germany from 2000 Hitachi 917 auto analyze, Roche Diagnostic, Swizterland). NonHDL-C was calculated as total-C minus HDL-C. The average of three systolic and diastolic blood pressure readings taken after a 2 min rest by an automatic device (DINAMAP, Criticon, Tampa, FL, USA) was used in analyses. Enlarged waist was defined as having a waist circumference $>102 \mathrm{~cm}$ for men and $>88 \mathrm{~cm}$ for women [21].

\section{Statistical Analyses}

Baseline demographic characteristics were evaluated for men and women using X2 and t-tests for differences in proportions and means comparing those with and without an enlarged waist circumference. Sex-specific quartiles of triglycerides were created to facilitate presentation of data. Previously we reported the cardiovascular risk factor levels by sex-specific triglyceride quartiles for men and women in the larger cohort in which we found that all risk factors were more adverse with increasing triglyceride quartiles: as the results were similar when restricted to those with an available waist circumference measure, these data are not presented in the current report [18].

AMI rates per 10,000 person-years were age-adjusted based upon 5-year age intervals, using the study's age distribution as the standard population. Age-adjusted rates of AMI were compared between those with and without an enlarged waist within each triglyceride quartile.
Age-adjusted and multivariable Cox proportional hazard analyses were conducted separately for men and women and results presented as Hazard Ratios and 95\% confidence intervals (CI). Proportional hazard assumption was evaluated by Schoenfeld's test. An elevated waist circumference (yes vs. no) by triglyceride level interaction term was tested in age and multivariable adjusted analyses where triglyceride levels were evaluated using several approaches: as $\mathrm{mmol} / \mathrm{L}$, sex-specific quartiles, and as high vs. low ( $<1.7$ vs. $>1.7 \mathrm{mmol} / \mathrm{L})$. We conducted stratified analyses in which we evaluated adjusted HRs by stratum of enlarged waist (yes vs. no) and elevated triglyceride (>1.7 mmol/L). We also conducted the above mentioned analyses using a triglyceride cut-off of $>2.0 \mathrm{mmol} / \mathrm{L}$, but as all results were similar they are not presented.

Multivariable models considered time since last meal (hrs) and conventional risk factors at baseline: age (yrs), current daily smoking (yes vs. no), systolic blood pressure $(\mathrm{mm} \mathrm{Hg})$, self-reported diabetes (yes vs. no), HDL-C and nonHDL-C ( $\mathrm{mmol} / \mathrm{L})$, and frequency of alcohol consumption ( $>$ once a week, once per week, two-three times per month, once per month or less). The percentage of excess risk associated with the hypertriglyceridemic-waist phenotype that was mediated by HDL-C and nonHDL-C was evaluated as follows:

[ (HR (smoking and age adjusted) - HR (smoking, age, HDL-C and nonHDL-C adjusted))/HR (smoking and age adjusted)-1 ] x 100 [22].

Significance was determined by $\mathrm{P}<0.05$. Stata 12 (StataCorp LP, College Station, Texas) was used in all analyses.

\section{Results}

At baseline, the average age was 47.4 yrs $(\mathrm{SD}=14.3)$ for men and 46.3 yrs $(S D=14.1)$ for women and an enlarged waist was present for $13 \%$ of men and $21.5 \%$ of women. For men and women, those with an enlarged waist were, on average, 5-6 years older, more likely to have diabetes, and less likely to be smokers or to have any college education (Table 1). All baseline cardiovascular risk factors were more adverse among those with an enlarged waist (all P-values $<0.0001$ ). The time since last meal ranged from 0 to 8 hours in which $84.8 \%$ had a time since last meal of 4 hours or less. For each quartile and decile of triglycerides, the median time since last meal was 2 hours (IQR was 1-3 hours).

The mean follow-up time was 11.5 yrs $(\mathrm{SD}=2.8)$ for both men and women. A total of 2,537 (4.6\%) men and 1,086 (1.8\%) women developed an AMI. In stratified analyses in which normal and elevated triglyceride levels were evaluated by the presence and absence of an enlarged waist, we observed that the HRs associated with the presence of both conditions in men (who had a $68 \%$ excess risk) and women (who had a 95\% excess risk) reflected what would be expected assuming an additive effect of having an enlarged waist and an elevated triglyceride level (model 1, Table 2). After further adjustment for HDL and nonHDL-C (model 2), the HR were considerably attenuated with $75 \%$ of the excess risk associated with the phenotype being mediated by HDL-C and nonHDL-C. With adjustment for additional risk factors, no significant association with AMI was observed for elevated triglycerides, enlarged waist or for having both conditions vs. none (model 3, Table 2). Further, there was no significant enlarged waist circumference (yes vs. no) by triglyceride multiplicative interaction term in age-adjusted or multivariable adjusted analyses in men and women.

In age-adjusted analyses (not presented in the tables), an enlarged waist circumference was associated with greater age-adjusted HRs for 
Citation: Egeland GM, Igland J, Nygard O, Sulo G, Tell GS (2015) Hypertriglyceridemic-Waist Phenotype is a Useful Global Assessment Tool for Predicting Acute Myocardial Infarction. J Cardiovasc Dis Diagn 3: 207. doi:10.4172/2329-9517.1000207

Page 3 of 6

\begin{tabular}{|c|c|c|c|c|}
\hline & \multicolumn{2}{|c|}{ MEN } & \multicolumn{2}{|c|}{ WOMEN } \\
\hline & \multicolumn{2}{|c|}{ Waist } & \multicolumn{2}{|c|}{ Waist } \\
\hline & Normal & Enlarged $^{b, c}$ & Normal & Enlarged $^{\mathrm{b}, \mathrm{c}}$ \\
\hline No. Participants & 48,309 & 7,251 & 47,556 & 12,995 \\
\hline Age (yrs) & $47.2(14.3)$ & $52.2(14.6)$ & $45.4(13.5)$ & $51.0(14.7)$ \\
\hline nonHDL-C (mmol/L) & $4.44(1.13)$ & $4.91(1.07)$ & $4.00(1.16)$ & $4.73(1.21)$ \\
\hline HDL-C (mmol/L) & $1.27(0.34)$ & $1.13(0.30)$ & $1.55(0.39)$ & $1.35(0.35)$ \\
\hline Triglycerides (mmol/L) & $1.86(1.20)$ & $2.55(1.56)$ & $1.25(0.75)$ & $1.93(1.13)$ \\
\hline$\%$ Diabetes & 1.5 & 3.6 & 0.8 & 3.3 \\
\hline Glucose $(\mathrm{mmol} / \mathrm{L})$ & $5.36(1.32)$ & $5.78(1.81)$ & $5.11(1.00)$ & $5.52(1.59)$ \\
\hline$\%$ Current smokers & 31 & 28 & 33 & 29 \\
\hline BMI $\left(\mathrm{kg} / \mathrm{m}^{2}\right)$ & $25.5(2.7)$ & $31.3(3.3)$ & $23.9(2.9)$ & $31.0(4.1)$ \\
\hline Waist circumference $(\mathrm{cm})$ & $89(6.9)$ & $108(6.4)$ & $76(6.5)$ & $96(7.6)$ \\
\hline $\mathrm{SBP}(\mathrm{mm} \mathrm{Hg})$ & $134(16.5)$ & $141(18.2)$ & $125(18.4)$ & $137(21.6)$ \\
\hline $\mathrm{DBP}(\mathrm{mm} \mathrm{Hg})$ & $78(10.6)$ & $83(13.1)$ & $73(10.7)$ & $78(11.7)$ \\
\hline$\%$ any college education & 32 & 25 & 33 & 21 \\
\hline \multicolumn{5}{|c|}{$\begin{array}{l}\text { aData presented as means (SD) unless designated as percent (\%) } \\
\text { befined as }>102 \mathrm{~cm} \text { for men and }>88 \mathrm{~cm} \text { for women } \\
\text { 'Differences in all characteristics presented between normal and enlarged waist circumference groups are statistically significant with P < } 0.0001 \\
\text { Abbreviations: nonHDL-C: nonhigh-density-lipoprotein cholesterol; HDL-C: high-density-lipoprotein cholesterol; BMI: body mass index; SBP and DBP: systolic and } \\
\text { diastolic blood pressure, respectively. }\end{array}$} \\
\hline
\end{tabular}

Table 1: Baseline characteristics of study population by presence or absence of an enlarged waist circumference: Cohort Norway ${ }^{\text {. }}$

\begin{tabular}{|c|c|c|c|c|}
\hline & \multicolumn{2}{|c|}{ Normal Waist } & \multicolumn{2}{|c|}{ Enlarged Waist ${ }^{a}$} \\
\hline & $\begin{array}{l}\text { Low Triglyceride } \\
<1.7 \mathrm{mmol} / \mathrm{L}\end{array}$ & $\begin{array}{c}\text { Elevated Triglyceride } \\
\geq 1.7 \mathrm{mmol} / \mathrm{L}\end{array}$ & $\begin{array}{l}\text { Low Triglyceride } \\
<1.7 \mathrm{mmol} / \mathrm{L}\end{array}$ & $\begin{array}{c}\text { Elevated Triglyceride } \\
\geq 1.7 \mathrm{mmol} / \mathrm{L}\end{array}$ \\
\hline $\begin{array}{c}\text { MEN } \\
\text { No. of Participants } \\
\text { No. of cases }\end{array}$ & $\begin{array}{c}27,049 \\
983\end{array}$ & $\begin{array}{c}21,259 \\
1,081\end{array}$ & $\begin{array}{c}2,267 \\
130\end{array}$ & $\begin{array}{c}4,984 \\
343\end{array}$ \\
\hline $\mathrm{HR}$, model $1^{\mathrm{b}}$ & 1.0 & $1.45(1.32-1.58)$ & $1.16(0.95-1.41)$ & $1.68(1.48-1.90)$ \\
\hline $\mathrm{HR}$, model $2^{\mathrm{c}}$ & 1.0 & $1.09(0.98-1.20)$ & $1.05(0.86-1.27)$ & $1.17(1.02-1.35)$ \\
\hline HR, model $3^{d}$ & 1.0 & $1.07(0.96-1.19)$ & $1.01(0.82-1.25)$ & $1.14(0.99-1.32)$ \\
\hline $\begin{array}{l}\text { WOMEN } \\
\text { No. of Participants } \\
\text { No. of cases }\end{array}$ & $\begin{array}{c}38,959 \\
405\end{array}$ & $\begin{array}{c}8,597 \\
272\end{array}$ & $\begin{array}{c}6,639 \\
136\end{array}$ & $\begin{array}{c}6,356 \\
273\end{array}$ \\
\hline $\mathrm{HR}$, model $1^{\mathrm{b}}$ & 1.0 & $1.55(1.32-1.82)$ & $1.30(1.06-1.59)$ & $1.95(1.66-2.29)$ \\
\hline $\mathrm{HR}$, model $2^{\mathrm{c}}$ & 1.0 & $1.03(0.86-1.23)$ & $1.16(0.95-1.43)$ & $1.24(1.03-1.49)$ \\
\hline HR, model $3^{d}$ & 1.0 & $1.06(0.86-1.30)$ & $1.12(0.88-1.43)$ & $1.11(0.89-1.39)$ \\
\hline
\end{tabular}

a for men $>=102 \mathrm{~cm}$ for women $>=88 \mathrm{~cm}$

${ }^{\mathrm{b}}$ model 1: baseline age (yrs), current daily smoking (yes vs. no), time since last meal (hrs).

${ }^{c}$ model 1 plus HDL-C and nonHDL-C ( $\left.\mathrm{mmol} / \mathrm{L}\right)$.

d model 1 plus HDL-C and nonHDL-C (mmol/L) and systolic blood pressure (mmHg), self-reported diabetes (yes vs. no), and frequency of alcohol consumption in past year (>1 week, 1 per week, 2-3 times per month, 1 per month or less).

Table 2: Age and multivariable adjusted hazard ratios $(95 \% \mathrm{Cl})$ for incident acute myocardial infarction by the presence or absence of an enlarged waist and elevated triglyceride level:Cohort Norway.

AMI for both men $(\mathrm{HR}=1.2 ; 95 \% \mathrm{CI} 1.1-1.4)$ and women $(\mathrm{HR}=1.4$; $95 \%$ CI 1.2 - 1.6). However, an enlarged waist did not predict AMI in multivariable models considering conventional risk factors in men (HR $=1.0 ; 95 \% \mathrm{CI} 0.9-1.1)$ or in women $(\mathrm{HR}=1.1 ; 95 \% \mathrm{CI}=0.9-1.2)$. Further, in analyses stratified by sex-specific quartiles of triglycerides, only modest additional risk was associated with an enlarged waist circumference in men and women (Figure 1). To illustrate, for men in the highest triglyceride quartile $(>2.33 \mathrm{mmol} / \mathrm{L})$, age-adjusted AMI rates were 58.4 and 51.1 per 10,000 person years for those with and without an enlarged waist, respectively ( $\mathrm{HR}=1.1 ; 95 \% 0.9-1.3)$ (Figure 1). In women in the highest sex-specific triglyceride quartile (>1.67 mmol/L), age-adjusted AMI rates were 25.6 and 21.9 per 10,000 person years for those with and without an enlarged waist, respectively $(\mathrm{HR}=1.17 ; 95 \% \mathrm{CI}=1.04-1.45, \mathrm{P}=0.02)$. These differences were no longer significant after considering conventional risk factors. However, we do note wide confidence intervals with increasing triglyceride quartiles for women with an enlarged waist (Figure 1).

\section{Conclusions}

The presence of the hypertriglyceridemic-waist phenotype was associated with increased risk of AMI with stronger associations noted for women than for men. We found that the majority of excess risk associated with having both conditions, compared to neither condition, was mediated through HDL-C and nonHDL-C. HDL-C and nonHDL-C are both highly related to triglyceride levels and to an enlarged waist circumference [17]. Also, HDL-C and nonHDL-C provide important information about underlying lipoprotein metabolism in which the need for additional consideration of triglyceride levels becomes less important [23]. Further, when additional risk factors were considered, the phenotype and stratum-specific combinations of enlarged waist and elevated triglycerides were of no value in the prediction of AMI.

\section{Strengths and Limitations}

Strengths of our study include the large community-dwelling 

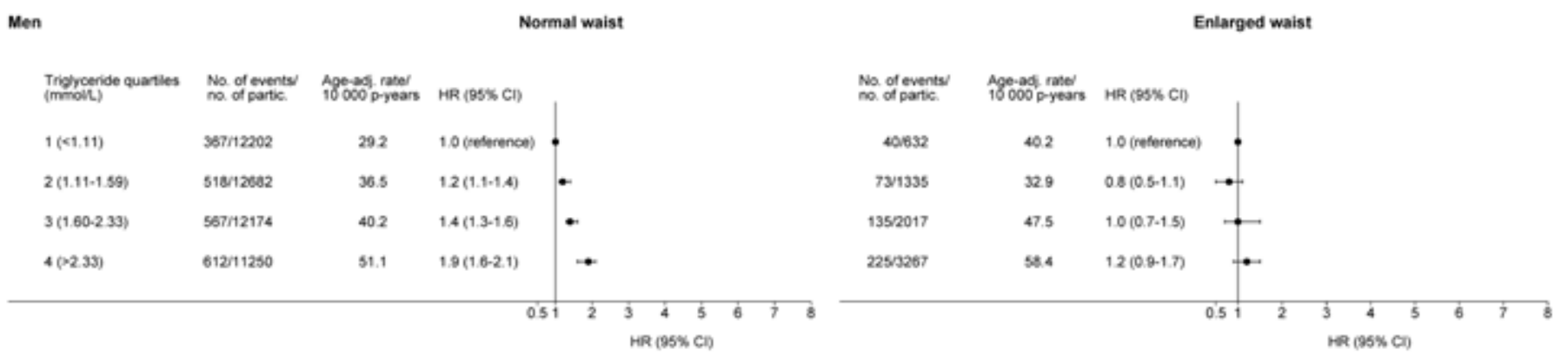

Women
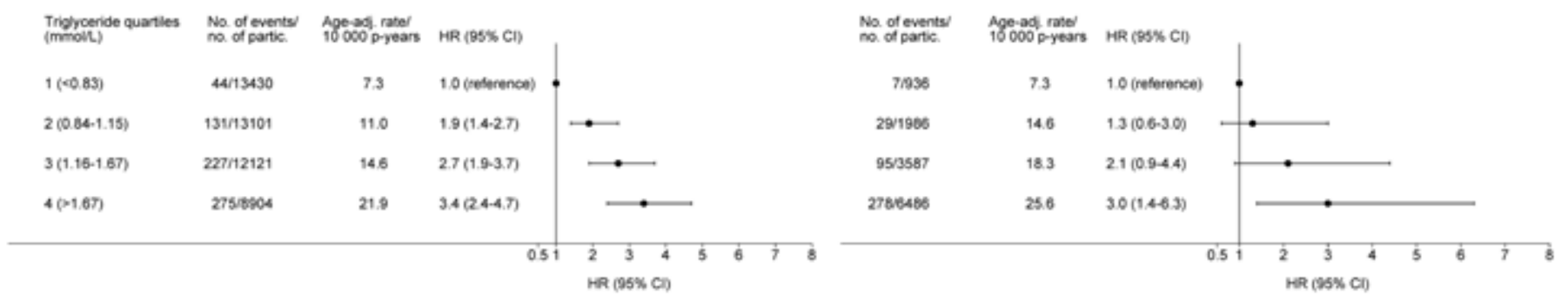

Figure 1: Age-adjusted hazard ratios (HR) and 95\% confidence intervals $(\mathrm{Cl})$ for incident acute myocardial infarction by non-fasting triglyceride quartiles and an enlarged waist circumference: Cohort Norway.

cohort and the assessment of nonfasting triglyceride levels which is now considered superior to fasting triglycerides in predicting coronary heart disease [13]. Also, while we were not able to adjust for LDL-C, we did include nonHDL-C and HDL-C in our multivariable models, which is another strength of the study given that nonHDL-C may be a stronger risk factor than LDL-C in risk prediction and that there is evidence that HDL-C and nonHDL-C, or the ratio of total cholesterol to HDL-C, are more closely related than LDL-C to the atherogenic dyslipidemia observed in obesity [23-26]. Another important strength is the comprehensive ascertainment of follow-up case status through record linkages with hospital discharge diagnoses and the Cause of Death Registry. Given the homogenous ethnic Norwegian study population, one limitation is that our results are not generalizable to other ethnic/racial groups.

Results of other prospective studies have provided mixed results, likely reflecting differences in definitions of the phenotype, characteristics of the population studied, sample size, and the extent to which analyses adjusted for important covariates [8-12]. In a cohort of 557 postmenopausal Danish women followed for 8.5 years, the presence of both an enlarged waist $(>88 \mathrm{~cm})$ and elevated fasting triglycerides $(>1.45 \mathrm{mmol} / \mathrm{L}$ ), when compared to those without the phenotype, conveyed a 4.7 -fold increased risk for CVD mortality based upon 36 deaths, adjusting for age, smoking, and low-density lipoprotein cholesterol [11]. In the Tehran Lipid and Glucose study, 6,834 men and women were followed for an average of 9.3 years in which the presence of the phenotype significantly predicted incident CVD among women (HR of 1.47, 95\% CI 1.11-1.94), but not men, in analyses adjusting for a number of risk factors including the Framingham risk score [10]. In the EPIC-Norfolk Prospective Population Study of 21,787 participants followed for a mean of 9.8 years, sex differences were noted in the relative importance of the phenotype in predicting coronary artery disease [8]. In women, but not men, those with both an enlarged waist and elevated triglyceride level were at significantly greater risk for incident CVD (adjusted HR of 1.67) when compared separately to all other strata of enlarged waist with or without an elevated triglyceride level. However, in additional analyses considering the Framingham risk score, the presence of the phenotype significantly predicted coronary artery disease only among those in the lowest risk tertile of the Framingham risk score [8]. In 3,430 male participants of an intervention trial of antioxidant supplementation (SU.VI.MAX study) prospectively followed for a mean of 7.5 years, the phenotype of an enlarged waist and elevated triglyceride level conveyed a 2.1-fold increased risk for incident CVD among men when compared to those with normal waist circumference and triglyceride levels, adjusting for age, smoking, blood pressure, physical activity and fasting glucose, but not lipids [9]. In the large population-based Chinese cohort (the Kailuan Study) of 76,605 men and 19,410 women with a mean followup period of 4 years, the presence of the phenotype ( $>90 \mathrm{~cm}$ waist and $>2.0 \mathrm{mmol} / \mathrm{L}$ fasting triglyceride and $>85 \mathrm{~cm}$ waist and $>1.5 \mathrm{mmol} / \mathrm{L}$ triglyceride for men and women, respectively) was associated with a HR of 1.24 (95\% CI 1.07-1.44) for CVD after adjusting for conventional risk factors [12]. In a 5 year follow-up of 5,924 cardiovascular patients, evaluating the lipid accumulation product based upon triglyceride and waist circumference measures, unadjusted analyses identified the phenotype to be a significant predictor of mortality in women but not men; but results were attenuated and not significant in analyses adjusting for other risk factors [27].

Despite differences in study results, there is a tendency in the literature for an enlarged waist and an elevated triglyceride level to be a more important predictor of CVD in women than in men: findings which are compatible with our current study results. In a prior analysis of the same study population, we identified a stronger gradient in risk of AMI associated with deciles of nonfasting triglyceride values in women compared to men [18]. Also, we noted increasingly wider confidence intervals for HRs for AMI associated with increasing triglyceride quartiles for women with an at-risk waist circumference. Elevated 
Citation: Egeland GM, Igland J, Nygard O, Sulo G, Tell GS (2015) Hypertriglyceridemic-Waist Phenotype is a Useful Global Assessment Tool for Predicting Acute Myocardial Infarction. J Cardiovasc Dis Diagn 3: 207. doi:10.4172/2329-9517.1000207

triglyceride levels precede the development of diabetes, and central fat patterning associates with insulin resistance [28]. Also of note, is that diabetes is a stronger risk factor for CHD in women compared to men [29]. These considerations may explain the sex differences observed in the current study and in the literature.

Our findings do not support any additional value of incorporating assessment of an enlarged waist with an elevated nonfasting triglyceride value in $\mathrm{CHD}$ risk prediction when conventional risk factors are available. WHO's Expert Consultation on metabolic syndrome has emphasized its limited utility [30]. Indeed, with nearly three decades of research behind us, beginning with the related concept of Syndrome $\mathrm{X}$, which then led to the initial metabolic syndrome definition, the syndrome has not found its place in medical practice as a management or diagnostic tool [31]. Our current study evaluating the simple alternative to the metabolic syndrome, the hypertriglyceridemicwaist phenotype, suggests that it has no utility in terms of CHD risk prediction when conventional risk factors are available.

However, our findings do suggest that the phenotype is a useful global prediction tool, particularly for women. Patients presenting with the phenotype should be referred for standard clinical evaluation and treatment of CVD risk factors, as needed, and importantly they should be targeted with lifestyle interventions designed to increase physical activity and to improve dietary habits. The findings also suggest that further work is needed to explore the utility of the phenotype in CHD prediction in women given the wide confidence intervals for the HRs identified in women in the current study and the stronger associations observed for the phenotype in women compared to men in the literature.

\section{Conflicts of Interest}

The authors declare no conflicts of interest.

\section{Acknowledgements}

The authors thank Kari Juul, M.A., of CVDNOR for her administrative assistance and database management and Tomislav Dimoski, M.B.A., at the Norwegian Knowledge Centre for Health Services, Oslo, Norway for his contributions by developing software necessary for obtaining data from Norwegian hospitals, conducting the data collection and quality assurance of data in this project. KJ and TD were both compensated for their work. Funding was provided by the University of Bergen and the Norwegian Institute of Public Health.

\section{References}

1. Després JP, Lemieux I (2006) Abdominal obesity and metabolic syndrome. Nature 444: 881-887

2. Després JP, Cartier A, Côté M, Arsenault BJ (2008) The concept of cardiometabolic risk: Bridging the fields of diabetology and cardiology. Ann Med 40: 514-523.

3. Egeland GM, Cao Z, Young TK (2011) Hypertriglyceridemic-waist phenotype and glucose intolerance among Canadian Inuit: the International Polar Year Inuit Health Survey for Adults 2007-2008. CMAJ 183: E553-558.

4. Daniel M, Paquet C, Kelly SJ, Zang G, Rowley KG, et al. (2013) Hypertriglyceridemic waist and newly-diagnosed diabetes among remotedwelling Indigenous Australians. Ann Hum Biol 40: 496-504.

5. Pollex RL, Hanley AJ, Zinman B, Harris SB, Hegele RA (2006) Clinical and genetic associations with hypertriglyceridemic waist in a Canadian aboriginal population. Int J Obes (Lond) 30: 484-491.

6. He S, Zheng Y, Shu Y, He J, Wang Y, et al. (2013) Hypertriglyceridemic waist might be an alternative to metabolic syndrome for predicting future diabetes mellitus. PLoS One 8: e73292.

7. Brisson D, Perron P, Guay SP, Gaudet D, Bouchard L (2010) The "hypertriglyceridemic waist" phenotype and glucose intolerance in pregnancy. CMAJ 182: E722-725.
8. Arsenault BJ, Lemieux I, Després JP, Wareham NJ, Kastelein JJ, et al. (2010) The hypertriglyceridemic-waist phenotype and the risk of coronary artery disease: results from the EPIC-Norfolk prospective population study. CMAJ 182: $1427-1432$

9. Czernichow S, Bruckert E, Bertrais S, Galan P, Hercberg S, et al. (2007) Hypertriglyceridemic waist and 7.5-year prospective risk of cardiovascular disease in asymptomatic middle-aged men. Int J Obes (Lond) 31: 791-796.

10. Samadi S, Bozorgmanesh M, Khalili D, Momenan A, Sheikholeslami F, et al (2013) Hypertriglyceridemic waist: the point of divergence for prediction of CVD vs. mortality: Tehran Lipid and Glucose Study. Int J Cardiol 165: 260-265.

11. Tankó LB, Bagger YZ, Qin G, Alexandersen P, Larsen PJ, et al. (2005) Enlarged waist combined with elevated triglycerides is a strong predictor of accelerated atherogenesis and related cardiovascular mortality in postmenopausal women. Circulation 111: 1883-1890.

12. Wang A, Li Z, Zhou Y, Wang C, Luo Y, et al. (2014) Hypertriglyceridemic wais phenotype and risk of cardiovascular diseases in China: Results from the Kailuan Study. Int J Cardiol 174: 106-109.

13. Nordestgaard BG, Benn M, Schnohr P, Tybjaerg-Hansen A (2007) Nonfasting triglycerides and risk of myocardial infarction, ischemic heart disease, and death in men and women. JAMA 298: 299-308.

14. Bansal S, Buring JE, Rifai N, Mora S, Sacks FM, et al. (2007) Fasting compared with nonfasting triglycerides and risk of cardiovascular events in women. JAMA 298: 309-316.

15. Zilversmit DB (1979) Atherogenesis: a postprandial phenomenon. Circulation 60: $473-485$

16. Kolovou GD, Anagnostopoulou KK, Daskalopoulou SS, Mikhailidis DP, Cokkinos DV (2005) Clinical relevance of postprandial lipaemia. Curr Med Chem 12: 1931-1945.

17. Naess O, Søgaard AJ, Arnesen E, Beckstrøm AC, Bjertness E, et al. (2008) Cohort profile: cohort of Norway (CONOR). Int J Epidemiol 37: 481-485.

18. Egeland GM, Igland J2, Sulo G2, Nygård O3, Ebbing M4, et al. (2015) Nonfasting triglycerides predict incident acute myocardial infarction among those with favourable HDL-cholesterol: Cohort Norway. Eur J Prev Cardiol 22: 872881.

19. Sulo E, Vollset SE, Nygård O, Sulo G, Igland J, et al. (2015) Trends in 28-day and 1-year mortality rates in patients hospitalized for a first acute myocardial infarction in Norway during 2001-2009: a "Cardiovascular disease in Norway" (CVDNOR) project. J Intern Med 277: 353-361.

20. Sulo G, Vollset SE2, Nygård O3, Igland J4, Egeland GM2, et al. (2014) Trends in acute myocardial infarction event rates and risk of recurrences after an incident event in Norway 1994 to 2009 (from a Cardiovascular Disease in Norway Project). Am J Cardiol 113: 1777-1781.

21. National Cholesterol Education Program (NCEP) Expert Panel on Detection, Evaluation, and Treatment of High Blood Cholesterol in Adults (Adult Treatment Panel III) (2002) Third Report of the National Cholesterol Education Program (NCEP) Expert Panel on Detection, Evaluation, and Treatment of High Blood Cholesterol in Adults (Adult Treatment Panel III) final report. Circulation 106: 3143-3421.

22. Lin DY, Fleming TR, De Gruttola V (1997) Estimating the proportion of treatment effect explained by a surrogate marker. Stat Med 16: 1515-1527.

23. Bosomworth NJ (2013) Approach to identifying and managing atherogenic dyslipidemia: a metabolic consequence of obesity and diabetes. Can Fam Physician 59: 1169-1180.

24. Boekholdt SM, Arsenault BJ, Mora S, Pedersen TR, LaRosa JC, et al. (2012) Association of LDL cholesterol, non-HDL cholesterol, and apolipoprotein B levels with risk of cardiovascular events among patients treated with statins: a meta-analysis. JAMA 307: 1302-1309.

25. Emerging Risk Factors Collaboration, Di Angelantonio E, Sarwar N, Perry $\mathrm{P}$, Kaptoge S, et al. (2009) Major lipids, apolipoproteins, and risk of vascular disease. JAMA 302: 1993-2000.

26. Ramjee V, Sperling LS, Jacobson TA (2011) Non-high-density lipoprotein cholesterol versus apolipoprotein $B$ in cardiovascular risk stratification: do the math. J Am Coll Cardiol 58: 457-463.

27. loachimescu AG, Brennan DM, Hoar BM, Hoogwerf BJ (2010) The lipid accumulation product and all-cause mortality in patients at high cardiovascular risk: a PreCIS database study. Obesity (Silver Spring) 18: 1836-1844. 
Citation: Egeland GM, Igland J, Nygard O, Sulo G, Tell GS (2015) Hypertriglyceridemic-Waist Phenotype is a Useful Global Assessment Tool for Predicting Acute Myocardial Infarction. J Cardiovasc Dis Diagn 3: 207. doi:10.4172/2329-9517.1000207

Page 6 of 6

28. Wilson PW, Meigs JB, Sullivan L, Fox CS, Nathan DM, et al. (2007) Prediction of incident diabetes mellitus in middle-aged adults: the Framingham Offspring Study. Arch Intern Med 167: 1068-1074.

29. Huxley R, Barzi F, Woodward M (2006) Excess risk of fatal coronary heart disease associated with diabetes in men and women: meta-analysis of 37 prospective cohort studies. BMJ 332: 73-78.
30. Simmons RK, Alberti KG, Gale EA, Colagiuri S, Tuomilehto J, et al. (2010) The metabolic syndrome: useful concept or clinical tool? Report of a WHO Expert Consultation. Diabetologia 53: 600-605.

31. Reaven GM (2011) The metabolic syndrome: time to get off the merry-goround? J Intern Med 269: 127-136. 